\title{
Drugs in the headlines
}

This year witnessed both surprising successes and unexpected failures in basic and clinical drug development. There were also mixed results for some newly tested drugs, which will probably prompt a careful reassessment of their therapeutic value. Our drug watch compilation summarizes the most talked about therapies of the year.

\section{GREEN LIGHT}

Olaparib and BSI-201

These compounds, which inhibit a DNA repair enzyme called poly-ADP-ribose polymerase (PARP), showed benefits in early clinical trials, including shrinkage of tumors lacking known drug targets, such as estrogen receptors.

\section{Rasagiline}

This drug, which inhibits the breakdown of dopamine and is currently used to ease symptoms of Parkinson's disease, got a major boost thanks to clinical trials that suggested it could substantially delay progression of the illness.

\section{Benlysta}

In the first ever phase 3 trial for end-stage lupus, this monoclonal antibody unexpectedly improved disease indicators, opening the way for long-awaited therapies.

\section{Glatiramer acetate (Copaxone)}

This synthetic amino acid polymer was previously employed for the treatment of relapsing-remitting multiple sclerosis. Its use has now been extended to treat early, isolated neurologic episodes associated with multiple sclerosis, thanks to its newly demonstrated efficiency in delaying attack onset.

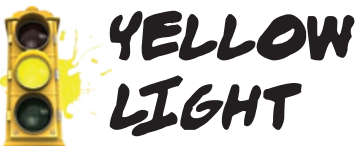

\section{Avastin}

This antibody, which blocks blood vessel growth, was approved in the US as a treatment for glioblastoma and renal cell carcinoma. However, it failed in trials against early-stage colon cancers. Reports have also suggested that targeting blood vessel growth factors might promote cancer metastasis in certain circumstances.

\section{Denosumab}

Several clinical trials tested this antibody, which counteracts bone loss. Although a US Food and Drug Administration committee recommended its approval for osteoporosis treatment, it recommended against approval of its preventive use-and its use to treat bone loss in certain cancer cases - until more data are available.

\section{GRNOPC1}

Regulators approved clinical trials of this human embryonic stem cell therapy in patients with spinal cord injury. But Geron, the company developing the treatment, temporarily delayed the trial, owing to the detection of microscopic spinal cysts in some rodent tests of the therapy.

\section{GDC-0449}

Clinical studies showed that this cell signaling inhibitor can slow the growth of aggressive brain tumors. This success, however, was followed by reports of drug resistance mutations in subjects, tempering hopes for broad anticancer use.

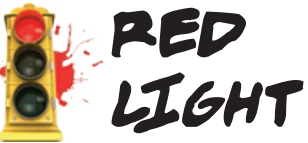

\section{Prochymal}

Despite receiving fast-tracked approval, this adult stem cell therapy did not yield the expected results in phase 3 trials against two types of severe graft-versus-host disease, and other prospective applications might also need reevaluation.

\section{Desmoteplase}

A thrombolytic agent derived from vampire bat saliva, this drug was purported as a treatment for acute ischemic stroke after the success of phase 2 testing. But the results of a phase 3 study showed no disease benefits, stalling further development.

\section{Pactimibe}

This treatment was intended to prevent atherosclerosis by inhibiting the buildup of cholesterol inside cells, but advanced clinical trials showed that it might actually promote symptoms and increase cardiovascular disease risk.

\section{Bextra}

Pfizer, the company manufacturing this controversial COX-2 inhibitor, reportedly agreed to pay $\$ 2.3$ billion to settle allegations that it illegally marketed this painkiller for unapproved uses.

Victoria Aranda, New York

Corrected after print 8 and 11 December 2009

\section{In case you missed it}

\section{A look at stories that fell under the radar in 2009}

The results of the first completed 'phase 0' trial in cancer therapy were announced. The phase 0 trial is the earliest clinical trial type in the US, designed to give preliminary data about the action and bioavailability of candidate medicines. The 2009 phase 0 trial in oncology came from Abbott Laboratories, which was exploring the potential of a poly-ADP-ribose polymerase inhibitor.

Cervarix received approval from the US Food and Drug Administration. This vaccine, from GlaxoSmithKline, blocks infection by certain strains of the human papillomavirus and thereby prevents against cervical cancer. The approval received little fanfare compared with that of Merck's Gardasil.
Mouse studies suggested new consequences of high-fat diets in moms-to-be. In research presented at the annual meeting of the Society for Neuroscience, scientists reported that mouse pups born to mothers fed a high-fat diet during pregnancy showed a greater affinity for drinking a sugar solution than their control counterparts. The finding comes at a time when type 2 diabetes is increasingly common, including among teens.

A retrovirus was found to be common in aggressive prostate cancers. An analysis of prostate cancer samples found that the presence of the Xenotropic murine leukemia virus-related virus (XMRV) - a common retrovirus - was associated with more aggressive tumors. In a study published shortly afterward, another team of scientists reported a possible link between XMRV and chronic fatigue syndrome. 


\section{ER R ATA}

\section{Erratum: Drugs in the headlines}

\section{Victoria Aranda}

Nat. Med. 15, 1353 (2009); published online 4 December 2009; corrected after print 8 December 2009

In the version of this article initially published, the sentence "However, it failed in trials against early-stage colon cancers, and reports suggested that it might promote cancer metastasis in certain circumstances" was incorrect. Although it has been suggested that other strategies targeting blood vessel growth factors might promote tumor growth, this has not been specifically shown for Avastin. The text should read "However, it failed in trials against early-stage colon cancers. Reports have also suggested that targeting blood vessel growth factors might promote cancer metastasis in certain circumstances." The error has been corrected in the HTML and PDF versions of the article.

\section{Erratum: Drugs in the headlines}

\section{Victoria Aranda}

Nat. Med. 15, 1353 (2009); published online 4 December 2009; corrected after print 8 December 2009; corrected after print 11 December 2009

In the version of this article initially published, it was stated that Denosumab had gained approval for use in osteoporosis treatment, when in fact it had only been recommended for approval. Additionally, the approval of its preventive use was recommended against until further data are available. The text should read "Several clinical trials tested this antibody, which counteracts bone loss. Although a US Food and Drug Administration committee recommended its approval for osteoporosis treatment, it recommended against approval of its preventive use-and its use to treat bone loss in certain cancer cases_ - until more data are available." The error has been corrected in the HTML and PDF versions of the article. 